\title{
Determination of the Velocity of Meteors Based on Sinodial Modulation and Frequency Analysis
}

\author{
Felix Bettonvil
}

Received: 1 July 2007/Accepted: 2 October 2007/Published online: 17 October 2007

(C) Springer Science+Business Media B.V. 2007

\begin{abstract}
In meteor photography the velocity of meteors is generally obtained from a chopper which blocks periodically the incident light beam in front of the camera lens. In this paper I examine modulation of the meteor trail instead with a sinodial function and use frequency analysis to compute accurately the mean atmospheric velocity.
\end{abstract}

Keywords Photography · Velocity · Orbital elements · High-precision · All-sky · Fireball patrol · Camera · Deceleration · Shutter · Fisheye lens

\section{Introduction}

In 2006 I described a digital All-sky camera for fireball patrol work based on a Nikon Coolpix 4500 camera with a FC-E8 fisheye converter lens (Bettonvil 2006a). The idea of using a digital camera for fireball work was based on the computation of a fireball trajectory from scanned photographic film with only medium resolution $(2722 \times 2338$ pixels, approx. 5'/pixel) which gave acceptable orbits (Bettonvil 2006b). The medium resolution affected in particular the accuracy of the velocity.

Commonly rotating shutters are used to measure the velocity, as already demonstrated by Elkin more then a century ago (Millman 1936). They consist of a wheel, rotating in front of the lens with a constant speed and having a number of segments which block the incident light beam periodically, imaging the meteor trail on the camera as a dashed line. The edges of the breaks form the measurement points. From the distances between them and the known number of revolutions per second of the shutter, the semi-instantaneous and mean angular velocity can be calculated as well as the atmospheric velocity expressed in $\mathrm{km} / \mathrm{s}$ in the case the meteor trail has been captured from two different observing locations.

F. Bettonvil (ه)

Astronomical Institute, Utrecht University, Princetonplein 5, 3584 CC Utrecht, The Netherlands e-mail: F.C.M.Bettonvil@astro.uu.nl

F. Bettonvil

Netherlands Foundation for Research in Astronomy (ASTRON), Dwingeloo, The Netherlands 
The 4-Megapixel camera has an image scale of $6^{\prime} /$ pixel. If we assume a typical angular velocity for the meteor of $10 \% \mathrm{~s}$ and a shutter speed of $10 \mathrm{breaks} / \mathrm{s}$, one period measures only 10 pixels; 5 for the blocked and 5 for the unblocked part, which makes accurate measurement of the edges rather difficult. The uncertainty which is typically obtained for the mean atmospheric velocity is in the order of a few percent.

\section{Sinodial Modulation}

In Bettonvil (2007) I described the idea of modulation of the meteor trail with a triangular or sinodial function for measurement of the mean velocity. Fourier analysis techniques can reveal the dominant modulation frequency and thus the velocity. The advantage is that all pixels contribute in the measurement and not only the pixels locating the shutter breaks. Persistent trains, slow variations in brightness and/or flares give only minor problems because of their different frequency regime. The method leads as well to better results in the case of fore-shortened trails because the merging of breaks does not harm much.

\section{Simulations}

Simulations have been done to study the method in more detail. Artificial meteors with trail lengths of 100 pixels (which are typical for the described camera) were created including variations in brightness, background signal, saturation and noise. They were modulated with a sinodial function and then 8-bit digitized. With a Fast Fourier transform the frequency spectrum was computed with wavelengths from 2 to 100 pixels. The strongest peak, representing the modulation, was easily detected. A value for this frequency was obtained by fitting a Gaussian function through the peak (Fig. 1) which has some width due to the fact that the data sample is not infinite and sample and shutter frequency generally not exactly match, but which has no major influence on the frequency. The obtained values differed $0.1-0.4 \%$ from the real modulation frequency. An estimation of the uncertainty was computed from the differences of the Gauss fit with the data points in the frequency spectrum. It gave values from 0.2 until $0.5 \%$, hence it was concluded that it is a good approximation for the error.

A further test was done with a real video meteor which showed breaks with a sinodialmodulated appearance and which had similar path length (Fig. 2) and which was approx.
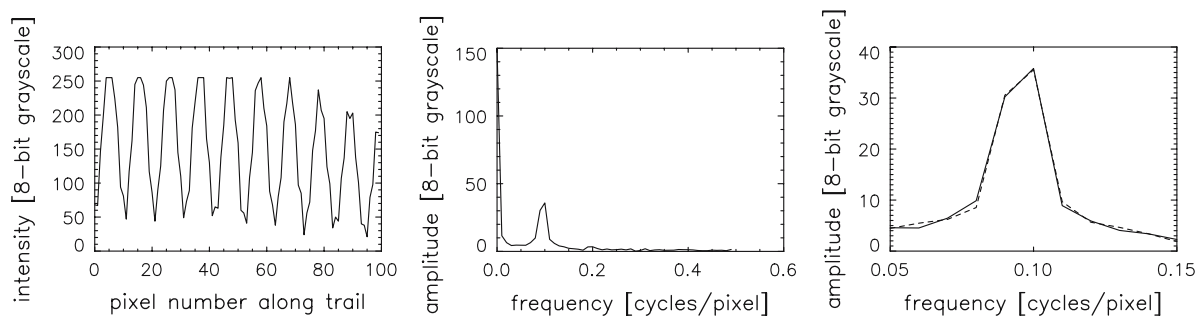

Fig. 1 A simulation of velocity determination of a meteor with sinodial modulation and FFT analysis. An artificial meteor trail is modulated with a sinodial function (left). Then the frequency spectrum is computed (center) which reveals clearly a peak around the modulation frequency. A Gaussian fit through the peak (right graph, dashed contour) is used to locate the peak frequency, which is in this case 0.096 cycles/pxl 

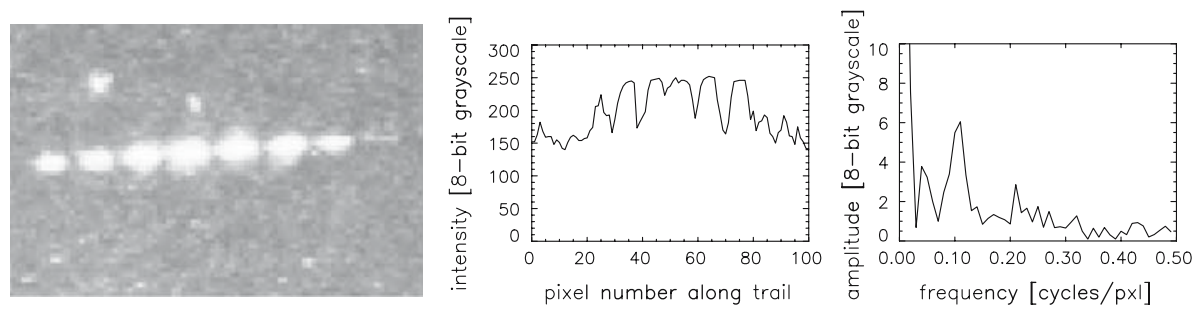

Fig. 2 Example of velocity determination with FFT analysis using real data: (left) image of a meteor trail taken with a video camera; (center) variation of the intensity along the trail as a function of pixel number; (right) frequency spectrum. The dominant frequency is 0.103 cycles/pxl, the computed uncertainty $0.5 \%$

perpendicular to the observer, hence with negligible foreshortening. The obtained uncertainty was of the same order.

As a final test, the trail was processed again but with the modulation information extracted in the conventional way by measuring the position of the begin point of every break and the frequency computed from their relative distances (including a weight factor which gave two neighboring points a lower weight than two points further apart). The obtained accuracy was $1.7 \%$; the difference with the FFT method $2 \%$.

\section{Test Setup}

Two prototypes of sinodial choppers have been made based on the idea described in Bettonvil (2007). The first is a conventional rotating shutter but consisting of many small blades with different size, sampling a sinodial function (Fig. 3, left). The second prototype modulates the light with two linear polarizers. One of them is fixed; the other rotating with a constant speed (Fig. 3, right). When the axes of linear polarization of the two polarizers are parallel, light is transmitted; when they are perpendicular, the light is blocked. The advantage of polarizers is that they give a continuous modulation, disadvantage that the total transmission is lower because of the use of only one linear polarization state. Moreover, meteors should not show polarization effects themselves.
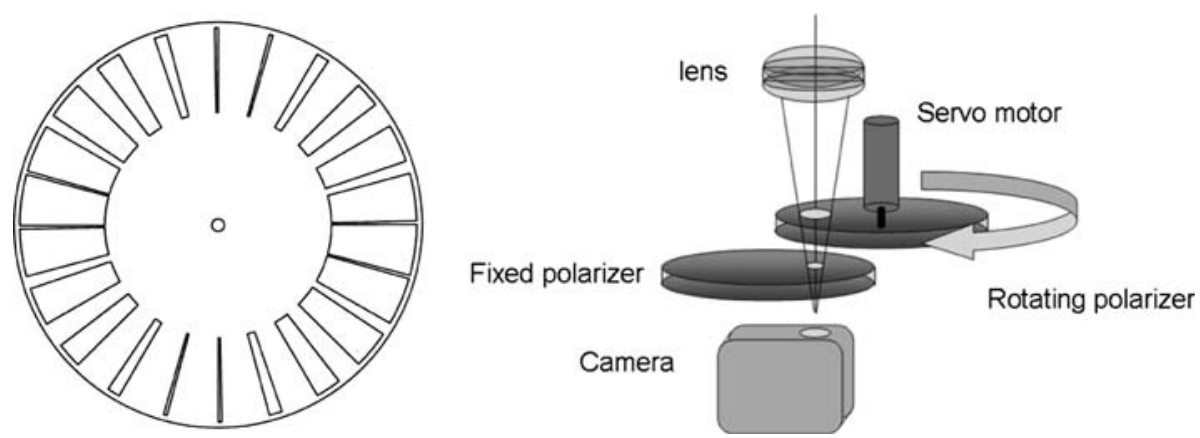

Fig. 3 Two alternatives for sinodial choppers: (left) mechanical shutter which is split up in 24 segments sampling a sinodial function; (right) use of two linear polarizers. One is fixed, the other rotates with a constant speed 


\section{Discussion and Conclusions}

It has been shown that sinodial modulation and frequency analysis are a good alternative for the common method of velocity determination and that it leads to a higher precision. It was found that fast modulations (30 cycles/s, the highest used in the simulations) gave the best accuracies.

The presented FFT analysis does, strictly spoken, not account for deceleration. Deceleration broadens the modulation peak in the frequency spectrum from which the mean velocity is computed. For fast meteors this is acceptable when calculating orbital elements (Betlem et al. 1999); for others and especially fireballs not. There we have to restrict ourselves to the first half of the trajectory which shows minimal deceleration in order to find more realistic pre-atmospheric velocities (Bettonvil 2006b). The FFT analysis however can be extended with Windowed Fourier Transformations and Wavelet analysis techniques which are capable of disentangling the deceleration. A quantitative study in terms of accuracy is foreseen.

Although invented for all-sky work, the presented method is naturally of advantage for cameras with (much larger) image scales too. With $10 \times$ longer focal lengths it would be realistic to obtain errors below $0.1 \%$ which is comparable with the best of other high precision orbit determination work (Betlem et al. 1999; Kohoutek 1959).

\section{References}

F. Bettonvil, Digital all-sky cameras II: a new method for velocity determination, in Proceedings of the International Meteor Conference 2006, ed. by F. Bettonvil, J. Kac (International Meteor Organization, Hove, 2007) in print

F. Bettonvil, A digital all-sky camera, in Proceedings of the International Meteor Conference 2005, ed. by L. Bastiaens, J. Verbert, J. Wislez, C. Verbeeck (International Meteor Organization, Hove, 2006a) pp. 90-98

F. Bettonvil, Orbit calculation of the August 15, 2002 fireball over the Netherlands, in Proceedings of the International Meteor Conference 2005, ed. by L. Bastiaens, J. Verbert, J. Wislez, C. Verbeeck (International Meteor Organization, Hove, 2006b) pp. 171-178

H. Betlem, J. Jenniskens, J. Van 't Leven, C. Ter Kuile, C. Johannink, H. Zhao, C. Lei, G. Li, J. Zhu, S. Evans, P. Spurný, Very precise orbits of 1998 Leonid meteors. Meteorit. Planet. Sci. 34, 979-986 (1999)

L. Kohoutek, On the precision of the photographical determination of the geocentric meteor velocity. Bull. Astron. Inst. Czech. 10, 120-134 (1959)

P. Millman, The importance of meteor photographs taken with a rotating shutter. J. R. Astron. Soc. Can. 30, 101-103 (1936) 